\title{
Regional Attenuation Correction of Weather Radar Using a Distributed Microwave-Links Network
}

\author{
Yang Xue, Xi-chuan Liu, Tai-chang Gao, Chang-ye Yang, and Kun Song \\ College of Meteorology and Oceanography, PLA University of Science and Technology, Nanjing, Jiangsu, China \\ Correspondence should be addressed to Xi-chuan Liu; liuxc85@gmail.com
}

Received 28 January 2017; Revised 11 April 2017; Accepted 27 April 2017; Published 22 May 2017

Academic Editor: Hongkai Gao

Copyright (C) 2017 Yang Xue et al. This is an open access article distributed under the Creative Commons Attribution License, which permits unrestricted use, distribution, and reproduction in any medium, provided the original work is properly cited.

The complex temporal-spatial variation of raindrop size distribution will affect the precision of precipitation quantitative estimates (QPE) produced from radar data, making it difficult to correct echo attenuation. Given the fact that microwave links can obtain the total path attenuation accurately, we introduce the concept of regional attenuation correction using a multiple-microwave-links network based on the tomographic reconstruction of attenuation coefficients. Derived from the radar-based equation, the effect of rainfall distribution on the propagation of radar and microwave link signals was analyzed. This article focuses on modeling of the tomographic reconstruction of attenuation coefficients and regional attenuation correction algorithms. Finally, a numerical simulation of regional attenuation correction was performed to verify the algorithms employed here. The results demonstrate that the correction coefficient $(0.9175)$ falls between the corrected and initial field of radar reflectivity factor (root mean square error, $2.3476 \mathrm{dBz}$; average deviation, $0.0113 \mathrm{dBz}$ ). Compared with uncorrected data, the accuracy of the corrected radar reflectivity factor was improved by $26.12 \%$, and the corrected rainfall intensity distribution was improved by $51.85 \%$ validating the region attenuation correction algorithm. This method can correct the regional attenuation of weather radar echo effectively and efficiently; it can be widely used for the radar attenuation correction and the promotion of quantitative precipitation estimation by weather radar.

\section{Introduction}

The attenuation of precipitation on the propagation of electromagnetic waves is one of the main factors that affect the quantitative precipitation estimation (QPE) by weather radar $[1,2]$. The attenuation effect will cause a decrease in radar echo intensity and detection area. In particular, at relatively far distances, radar reflectivity factors are lower than actual values and cannot reflect the actual distribution of rainfall. For short-wavelength radar, such as X-band radar, this type of attenuation is particularly serious. The development of correction techniques that address the attenuation of radar echoes has been extensively analyzed.

The most important aspect of attenuation correction is to obtain the characteristic of microwave attenuation. In some weather radar applications, researchers desire to measure attenuation along the propagation path to improve the effect of attenuation correction. Lin and Lv [3] proposed the use of a microwave radiometer to measure the total path attenuation. Serrar et al. [4] suggested the estimation of total attenuation of the echoes via the echo difference between sunny and rainy days. However, variations in terrain and fluctuations in the refraction in the path limit the usefulness of this method. Perez [5] introduced a method that can be used to obtain the attenuation of X-band radar by using dual-wavelength radar $(\mathrm{S}, \mathrm{X})$ based on the assumption that the S-band radar is with no attenuation. However, during relatively intense rainfall events, the attenuation difference between two bands is notably small and does not provide additional effective X-band attenuation information. The surface reference technique (SRT) is commonly used with space-borne precipitation radar to calculate the path attenuation by comparing the measured echo differences between areas of rainfall and nonrainfall. This method provides the advantage that the relative error decreases with an increase in integral path, but the reflection and attenuation of the electromagnetic waves are different because of the apparent fluctuation of the ground surface and humidity, which causes the SRT to have some limitations [6].

A new approach for rainfall measurement was recently presented using the attenuation caused by rainfall that affects the microwave signals for wireless data exchange $[7,8]$. 
Microwave propagation attenuation can be obtained by detecting the signal level of both the transmitter and receiver of a microwave link. During a rainfall event, the microwave is attenuated by falling raindrops when passing through the rain area. The degree of microwave attenuation can be obtained by measuring the difference between clear and rainy days, and these data can be used to retrieve rainfall intensity and its spatial distribution $[9,10]$ and correct the radar reflectivity factor as a constraint. Krämer et al. [11, 12] described forward and backward iterative algorithms that can be used to correct the X-band radar reflectivity factor using a $10.5 / 17.5 \mathrm{GHz}$ dual-frequency microwave link. Krämer and Verworn [13] used a dual-frequency microwave link to correct the C-band radar reflectivity factor. Cummings et al. [14] explored the use of two single-frequency microwave links in weather radar correction. However, these studies only enable corrections of the radar signal along the ray under the link path with a narrow range of a radial beam. Therefore, expanding the correction range using microwave links is worth exploring.

To improve the correction of radar attenuation by using microwave links, based on a microwave link for singlebeam correction, this paper proposes extending the region of radar attenuation correction using a multiple-microwave links network. In a radar detection area with simultaneously multiple communication links, several microwave links form a network that satisfies a certain topology. Deriving the attenuation coefficient of the grids based on the attenuation information of the microwave links network is the key to obtaining the total attenuation of each radial path of the radar as a reference. Therefore, in view of the above analysis, this paper presents a new method that is similar to that used in medical computerized tomography (CT) imaging technology. The area of radar and microwave link monitoring is addressed using discrete grids.

Section 2 of this paper analyzes the effects of rainfall distribution on the propagation of radar signals and microwave link signals. Section 3 analyzes and establishes the models of tomographic reconstruction of the attenuation coefficients while Section 4 proposes the regional attenuation correction algorithm, where a numerical simulation experiment spanning $20 \times 20 \mathrm{~km}$ was performed to verify the algorithm. Section 5 provides a summary and concluding remarks.

\section{Theory of Radar Echo Attenuation Correction}

When electromagnetic waves propagate in the atmosphere, the physical effects of weather target scattering, absorption, and reflection cause the energy of the propagation path to become attenuated. Suppose that $\bar{P}_{r 0}$ is the average echo power without considering the attenuation of the meteorological target and $\bar{P}_{r}$ is the average echo power after the attenuation is considered. The attenuation characteristic can be described by the attenuation factor $K_{r}$; then

$$
\bar{P}_{r}=\bar{P}_{r 0} \times K_{r} .
$$

Suppose $d \bar{P}_{r}$ is the attenuation value of the received power, which is affected by rainfall and other factors between radar and the target; this attenuation value can be expressed as

$$
d \bar{P}_{r}=-2 K_{t} \bar{P}_{r 0} d r .
$$

Next, the integral from 0 to $R$ is calculated, where $R$ is the distance between the radar and the target, and the average echo power is

$$
\bar{P}_{r}=\bar{P}_{r 0} \times e^{-2 \int_{0}^{R} K_{t} d r} .
$$

Therefore, the dimension of $K_{t}$ is $1 / \mathrm{km}$. Because the attenuation of the received power is usually expressed in decibels $(\mathrm{dB})$, the attenuation coefficient is converted into $k_{t}$ in $\mathrm{dB} / \mathrm{km}$. According to $\lg M=0.4343 \ln M$, (3) can be written as

$$
\ln \left(\frac{\bar{P}_{r}}{\bar{P}_{r 0}}\right)=-2 \int_{0}^{R} K_{t} d r
$$

Further derivation shows that

$$
\bar{P}_{r}=\bar{P}_{r 0} \times 10^{-0.2 \int_{0}^{R} k_{t} d r} .
$$

Next, compare (1) with (5)

$$
K_{r}=10^{-0.2 \int_{0}^{R} k_{t} d r} .
$$

If we use the radar reflectivity observation $Z_{m}\left(\mathrm{~mm}^{6} / \mathrm{m}^{3}\right)$ and the real radar reflectivity $Z_{r}\left(\mathrm{~mm}^{6} / \mathrm{m}^{3}\right)$ of the target instead of the echo power in the previous derivation process, then

$$
Z_{m}(r)=Z_{r}(r) \times K_{r}=Z_{r}(r) \times 10^{-0.2 \int_{0}^{R} k_{t} d r},
$$

where $R$ is the distance between the radar and the detection target, $K_{r}$ is the attenuation factor, and $k_{t}$ is the attenuation coefficient in $\mathrm{dB} / \mathrm{km}$. The logarithm of (7) is expressed as

$$
\lg Z_{m}=\lg Z_{r}-0.2 \int_{0}^{R} k_{t} d r
$$

In the process, we use the radar reflectivity factor. The conversion formula between radar reflectivity $Z\left(\mathrm{~mm}^{6} / \mathrm{m}^{3}\right)$ and radar reflectivity factor $z(\mathrm{dBz})$ is

$$
z=10 \log Z .
$$

Further derivation shows that

$$
z_{r}=z_{m}+2 \int_{0}^{R} k_{t} d r
$$

where $k_{t}$ is the attenuation coefficient in $\mathrm{dB} / \mathrm{km} ; 2 \int_{0}^{R} k_{t} d r$ is the total attenuation value of the radar path after integration in units of $\mathrm{dB} ; z_{r}$ is the corrected radar reflectivity factor, and $z_{m}$ is the radar-measured reflectivity factor. The accurate determination of the radar path integral attenuation is the key to attenuation correction. Therefore, using the attenuation of microwave link along the path att ${ }_{\text {rain }}$, the attenuation correction process can be expressed as

$$
z_{r}=z_{m}+2 \times \text { att }_{\text {rain }} .
$$




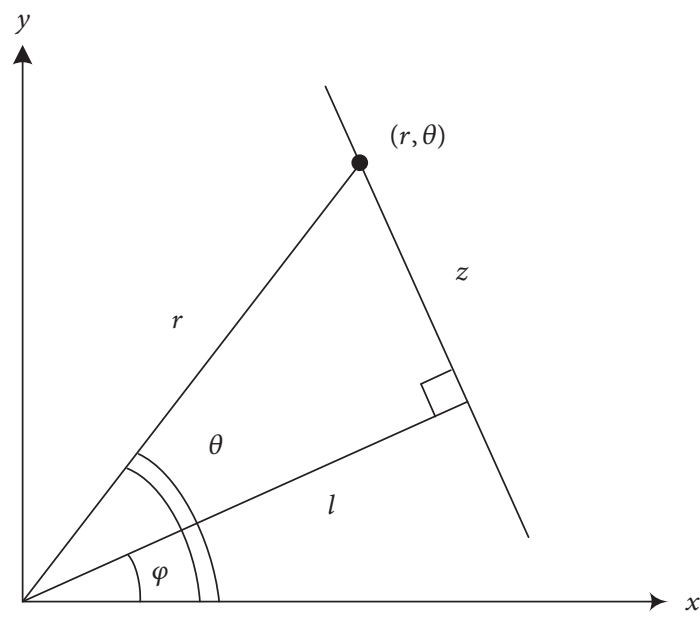

FIGURE 1: Coordinate system used in the Radon formula, where $r, z, l$ refer to the length, and $\theta, \phi$ refer to the angle.

\section{Tomographic Model of the Attenuation Coefficient}

3.1. Reconstruction of the Grid Attenuation Using Microwave Links. CT (computerized tomography) imaging technology is mainly based on ray scanning, where the attenuation of a ray power pass is obtained through the wave field. Tomographic techniques include techniques aimed toward reconstructing the cross-sectional distribution of a parameter from a set of one-dimensional transmission or reflection data, measured and collected along many different paths crossing the spatial domain where the object field has to be reconstructed. The theoretical basis of a CT image reconstruction algorithm is based on a Radon transform and Fourier slicing theorem [15]. This is characterized as follows: (1) the projection data contain the characteristic information of the original image, which can be reconstructed with the information; (2) in order to realize the reconstruction of the image, theoretically, an infinite number of continuous projection data is needed.

Radon transform is a linear integral projection transformation, and assumes the distribution function of a twodimensional target is $f(x, y)$; then Radon transform $p$ for the function $f(x, y)$ is integral along the straight line $z$ :

$$
\begin{aligned}
p & =\int_{-\infty}^{\infty} f(x, y) d z=\int_{-\infty}^{\infty} \widehat{f}(r, \theta) d z \\
& =\int_{-\infty}^{\infty} f\left(\sqrt{l^{2}+z^{2}}, \phi+\arctan \frac{z}{l}\right) d z .
\end{aligned}
$$

Therefore, the problem of tomographic image reconstruction involves the calculation of the image function $f(x, y)$ by the projection data $p$. The meanings of the other quantities in (12) are shown in Figure 1. Meanwhile, the Radon inverse transform satisfies

$$
\widehat{f}(r, \theta) d z=\frac{1}{2 \pi^{2}} \int_{0}^{\pi} \int_{-\infty}^{\infty} \frac{1}{r \cos (\theta-\phi)-l} \frac{\partial p}{\partial l} d l d \phi .
$$

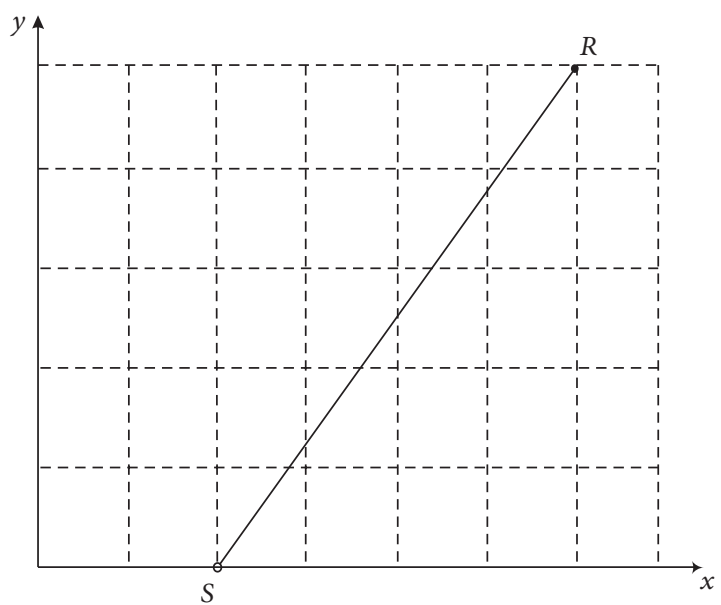

FIGURE 2: Reconstruction of the regional discrete diagram. This region was divided into $N$ equal square meshes $C_{j}(j=1, \ldots, N)$. A microwave link $L_{i}$ is taken as an example, where the transmitting and receiving terminals are $S$ and $R$, respectively.

The corresponding tomography is the inverse of the Radon transform. $f(x, y)$ is a continuous function, and the actual projection data are finite. Therefore, $f(x, y)$ as a solution is not unique. In practical work, a limited number of projection data is used to get a satisfactory reconstruction effect. Therefore, in the tomography, the continuous image is usually discretized to the pixel level initially, and the average value of $f(x, y)$ in each pixel is obtained.

With reference to the principle of CT imaging technology, the monitoring area is discretized. For the general measurement of the two-dimensional distribution, the nonuniform characteristic measurement area can be divided into $m \times n$ grids, with several imaging units (Figure 2). Assuming that the distribution within each grid is uniform, each grid cell can be referred to as a pixel. The $S$ site is a ray transmitter designed to emit with a fixed power, the $R$ site is an energy receiver designed to receive the ray energy after attenuation. The energy attenuation of the ray that passes through each grid is measured via the difference between the transmitted and received power. The pixel distribution $f(x, y)$ in the measurement area was reconstructed with the inversion technique.

The energy attenuation coefficient of each pixel is $\mathbf{K}(f(x$, $y)$ ), where the pixel-energy attenuation coefficient conversion operator is denoted as $\mathbf{K}(\cdot)$. Assuming that the propagation path of the $i$ th ray is $L_{i}$ and the total energy attenuation is $A_{i}$,

$$
A_{i}=\int_{L_{i}} \mathbf{K}(f(x, y)) d l
$$

The key to discrete image reconstruction is related to the problem of calculating the area attenuation coefficient vector $\mathbf{K}$ by measuring the total attenuation $A$ of the area through a series of rays.

3.2. Establishment of the Attenuation Coefficient Model. In the weather radar monitoring area, a network of microwave links pass through the rain area. The transmitting end of 


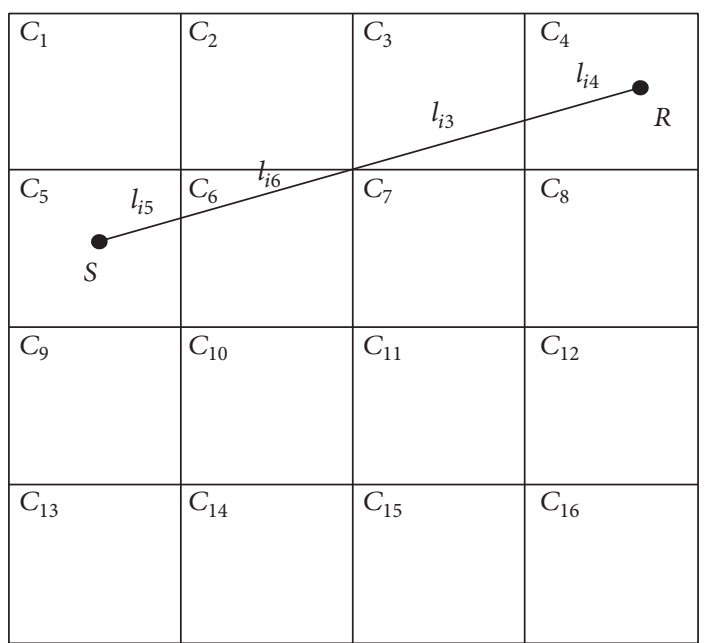

FIGURE 3: Schematic diagram of the chromatography. The link passes through the grid at $C_{5}, C_{6}, C_{3}$, and $C_{4}$, and their lengths in the grid are denoted as $l_{i 5}, l_{i 6}, l_{i 3}$, and $l_{i 4}$, respectively.

the microwave link realizes the transmission of a microwave signal with the fixed power, frequency, and polarization, and the receiving end receives the power after attenuation. Figure 3 provides a schematic representation of the attenuation coefficient tomography for a hypothetical region. This region was divided into $N$ equal square meshes $C_{j}(j=1, \ldots, N)$. A microwave link $L_{i}$ is taken as an example, where the transmitting and receiving terminals are $S$ and $R$, respectively. The link passes through the grid at $C_{5}, C_{6}, C_{3}$, and $C_{4}$, and their lengths in the grid are denoted as $l_{i 5}, l_{i 6}, l_{i 3}$, and $l_{i 4}$, respectively. In practical applications, the monitoring area was divided into the appropriate size and shape based on a combination of local geographical conditions, link density, and other information.

If the number of grids after discretization is $N$, the rain attenuation of the $i$ th microwave link can be expressed as

$$
A_{i}=\sum_{j=1}^{N} l_{i j} k_{j}
$$

where $l_{i j}$ is the length of the $i$ th microwave link that passes through the $j$ th grid; the unit of $k_{j}$ is $(\mathrm{dB} / \mathrm{km})$, and $i=1 \cdots M$ (for $M$ wireless microwave links).

According to the power law relation of rainfall attenuation, the rain attenuation of the link can be related to the link length, the average rain rate of the link, and the power law parameter of rain attenuation

$$
A_{i}=a_{i} R_{i}^{b_{i}} d_{i}
$$

where $A_{i}$ is the total attenuation of the propagation of the $i$ th microwave link; $R_{i}$ is the average observed rainfall intensity on the $i$ th link; $d_{i}$ is the length of the $i$ th link; $a_{i}$ and $b_{i}$ are the conversion constants of the $i$ th link.

Suppose that there are $M$ microwave links in the monitoring area. By combining (15), the rain attenuation of the $M$ microwave links can be expressed in vector form:

$$
L \mathbf{K}=A,
$$

where the total attenuation column vector is $A=\left(A_{i}\right)_{M \times 1}$; the distance matrix is $L=\left(l_{i j}\right)_{M \times N}$; the attenuation coefficient column vector is $\mathbf{K}=\left(k_{j}\right)_{N \times 1} ; k_{j}$ is the attenuation coefficient of the grid $C_{j}$ (assuming that the attenuation coefficient in the same grid cell is identical). $l_{i j}$ is the length of the $i$ th link in the grid $C_{j}$. Then, the chromatographic model can be further established as follows:

$$
\begin{gathered}
l_{11} k_{1}+l_{12} k_{2}+\cdots+l_{1 j} k_{j}+\cdots+l_{1 N} k_{N}=A_{1}, \\
l_{21} k_{1}+l_{22} k_{2}+\cdots+l_{2 j} k_{j}+\cdots+l_{2 N} k_{N}=A_{2}, \\
\vdots \\
l_{i 1} k_{1}+l_{i 2} k_{2}+\cdots+l_{i j} k_{j}+\cdots+l_{i N} k_{N}=A_{i}, \\
\vdots \\
l_{M 1} k_{1}+l_{M 2} k_{2}+\cdots+l_{i j} k_{j}+\cdots+l_{M N} k_{N}=A_{M} .
\end{gathered}
$$

3.3. Solving the Algorithm of the Attenuation Coefficient Model. The solution of the tomography model for attenuation coefficients is found by solving the linear system (18). Because the microwave links only pass through a small number of $N$ grids, their distribution is sparse with respect to the monitoring area. The number of unknown parameters is much larger than the number of equations. Therefore, it is an ill-posed linear inverse problem if one attempts to use the attenuation information of the microwave link to perform the attenuation coefficient tomographic inversion.

According to the characteristics of the linear equation, the attenuation coefficient field is iteratively solved. The related basic theory was recently explained and described in detail by Giuli et al. $[16,17]$ who provide more detailed information. To minimize the error of (17), the Simultaneous Iterative Reconstruction Technique was used to construct the cost function using the least-square criterion. The objective function was minimized by repeated iterations, and the optimal solution was obtained:

$$
J(\mathbf{K})=(A-L \mathbf{K})^{T}(A-L \mathbf{K})=\min ! .
$$

To obtain the minimum cost function,

$$
L^{T} A=L^{T} L \mathbf{K},
$$

the iterative formula needed to solve (18) can be written as

$$
\begin{aligned}
k_{j}(t) & \\
= & k_{j}(t-1) \\
& \quad+\frac{1}{M} \sum_{i=1}^{N} \frac{\left(A_{i}(t)-\sum_{p=1}^{M} l_{i p}(t) k_{p}(t-1)\right) \cdot l_{i j}(t)}{\sum_{p=1}^{M} l_{i p}(t)^{2}} .
\end{aligned}
$$

Here is the process of the specific iterative algorithm. First, let $t=0$ and give the initial value of the vector. Then, let $t=t+1$ and estimate $k(t)$ by (21). Finally, stop the iteration when $\|k(t)-k(t-1)\|<\varepsilon$ or $t>T$, where $\varepsilon, T$ is a predefined threshold. The process is repeated until the optimal solution is iterated. 


\section{Region Attenuation Correction Model and Numerical Simulation}

4.1. Region Attenuation Correction Model. Based on the establishment of the tomographic model for attenuation coefficients, the processing of the regional attenuation correction model (with the microwave link uniform distribution as an example) is described as follows:

(1) Construct a joint observation network of microwave links and weather radar data.

(2) The observational radar data and microwave attenuation data are matched with space and time, and the area is discretized.

(3) Using the rain attenuation of the microwave link network, a joint iterative reconstruction algorithm is used to obtain the rain attenuation coefficient $k_{j}$ of each grid cell.

(4) Assuming that there are $X$ lines between each grid point and the radar, we can substitute the attenuation coefficients obtained from (18) into (22). Then, we can get the path attenuation of the qth link between the radar and each grid point:

$$
\operatorname{Att}_{q j}=\sum_{q=1}^{X} \sum_{j=1}^{N} k_{j} \times d_{q j},
$$

where $d_{q j}$ is the length of the qth link between the radar and the grid point through the $j$ th grid.

(5) The corrected radar reflectivity factor $z_{q j}$ can be calculated by summing the radar-measured reflectivity factor $z_{m_{q j}}$ and total attenuation of the two-way path on the $q$ th link between the radar and the grid point:

$$
z_{q j}=z_{m_{q j}}+2 \times \mathrm{Att}_{q j}
$$

4.2. Establishment of the Initial Simulation Field. First, the 2D simulation region that corresponded to the microwave link and the radar data was established. Figure 4(a) shows the monitoring area of the microwave link network, which has a uniform distribution. The monitoring area was discretized, and the total distribution was $20 \times 20 \mathrm{~km}$, which was divided into many $2 \times 2 \mathrm{~km}$ square grids (Figure $4(\mathrm{~b})$ ). There were 34 microwave links in the simulation area. Among them, the microwave transmitting terminal is labeled as a point, the microwave receiving terminal is marked as pentagonal, the distance between adjacent transmitting and receiving terminals is $2 \mathrm{~km}$, and the density of the microwave link network is $0.17 / \mathrm{km}^{2}$. The microwave end transmitted the signal with a frequency of $9.47 \mathrm{GHz}$ and vertical polarization.

The initial field of rainfall had two weak-strong rainfall centers (Figure 5). Using the radar empirical relationship $Z=$ $300 R^{1.4}$ for simulation and calculation, the radar reflectivity factor initial field that corresponds to each grid was obtained (Figure 6).

The attenuation coefficient that corresponds to each grid was obtained through the $9.47 \mathrm{GHz}$ rain attenuation relationship $k_{o}=0.0085 R^{1.2645}$ in the International Telecommunication Union (ITU) rainfall attenuation model [18]. The attenuation of each grid was multiplied by the distance between the grid and the radar. The radar reflectivity factor observation field was gained by subtracting the initial field of the reflectivity factor from the attenuation that corresponds to each grid. Figure 7 shows the simulated radar reflectivity factor observation field.

4.3. Numerical Simulation Results and Analysis. To evaluate the correction algorithm, we used the correlation coefficient, mean square error, and average deviation to describe the correction effect:

$$
\begin{aligned}
\rho & =\frac{\sum_{j=1}^{N}\left(z_{r_{j}}-\overline{z_{r}}\right)\left(z_{j}-\bar{z}\right)}{\sqrt{\sum_{j=1}^{N}\left(z_{r_{j}}-\overline{z_{r}}\right)^{2} \sum_{j=1}^{N}\left(z_{j}-\bar{z}\right)^{2}}}, \\
\text { RMSE } & =\sqrt{\frac{1}{N} \sum_{j}^{N}\left(z_{r_{j}}-z_{j}\right)^{2}}, \\
e & =\frac{\sum_{j}^{N}\left(z_{j}-z_{r_{j}}\right)}{\sum_{j}^{N} z_{r_{j}}},
\end{aligned}
$$

where $z_{r}$ and $z$ are the initial and corrected radar reflectivity factor fields, respectively; $\overline{z_{r}}$ and $\bar{z}$ are the average initial and corrected radar reflectivity factors, respectively.

Figures 8(a) and 9(a) show the uncorrected radar reflectivity factor and uncorrected rainfall intensity, while Figures 8(b) and 9(b) show the corrected radar reflectivity factor and corrected rainfall intensity, respectively. Figures 8 and 9 show that the attenuation of the microwave link network significantly corrects the radar reflectivity factor and rainfall intensity. Compared with the uncorrected radar reflectivity factor, the correlation coefficient between the corrected reflectivity factor and the initial field of reflectivity factor improved from 0.4842 to 0.9175 . The mean square error decreased from 14.5655 to $2.3476 \mathrm{dBz}$, and the mean deviation decreased from 0.3011 to $0.0113 \mathrm{dBz}$. Compared with the uncorrected rainfall intensity, the correlation coefficient between the corrected rainfall intensity and the initial field of rainfall increased from 0.7315 to 0.9271 , the mean square error declined from 21.3271 to $8.2453 \mathrm{~mm} / \mathrm{h}$, and the mean deviation decreased from 0.7564 to $0.0359 \mathrm{~mm} / \mathrm{h}$. The corrected rainfall field can well reflect the position of the peak center, and the correction of the peak center of the heavy rainfall was slightly larger. The results show that the tomographic reconstruction model improved upon the radar echo attenuation correction quite well.

\section{Conclusions}

A regional attenuation correction method for weather radar is proposed based on using a multiple-microwave-links network using a tomographic reconstruction for attenuation coefficients. Derived from the radar equation, the effect of rainfall distribution on the propagation of the radar 


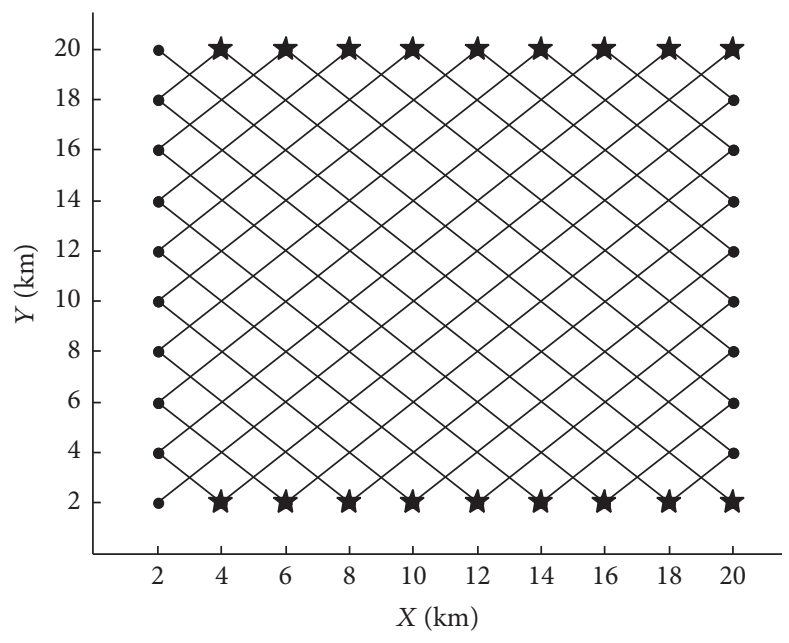

(a)

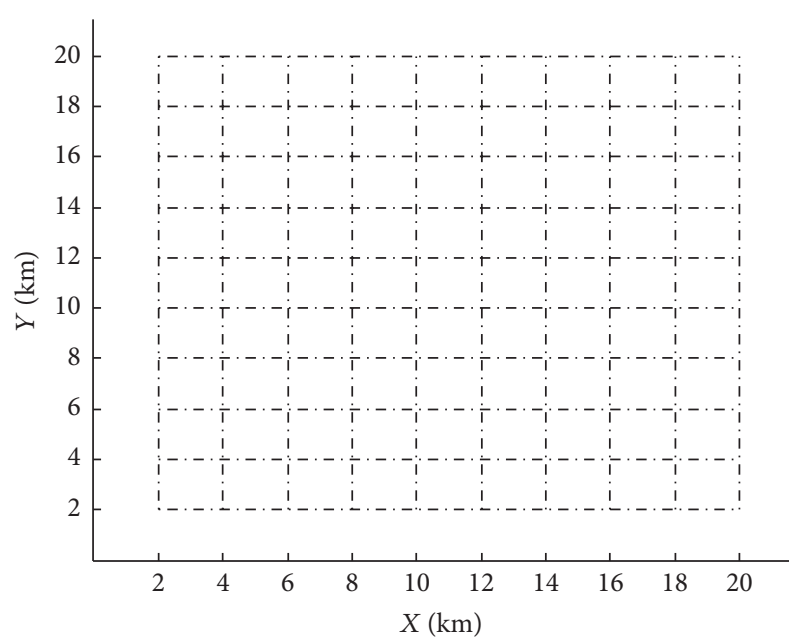

(b)

Figure 4: (a) The monitoring area of the microwave link network; (b) discretized monitor area. There were 34 microwave links in the simulation area. The monitoring area was divided into many $2 \times 2 \mathrm{~km}$ square grids.

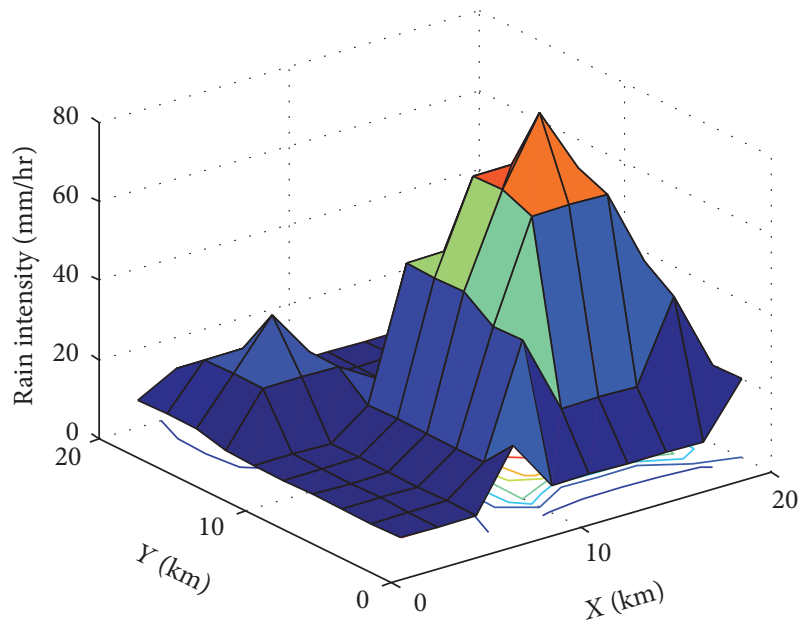

FIGURE 5: Initial field of rainfall intensity in the simulation area.

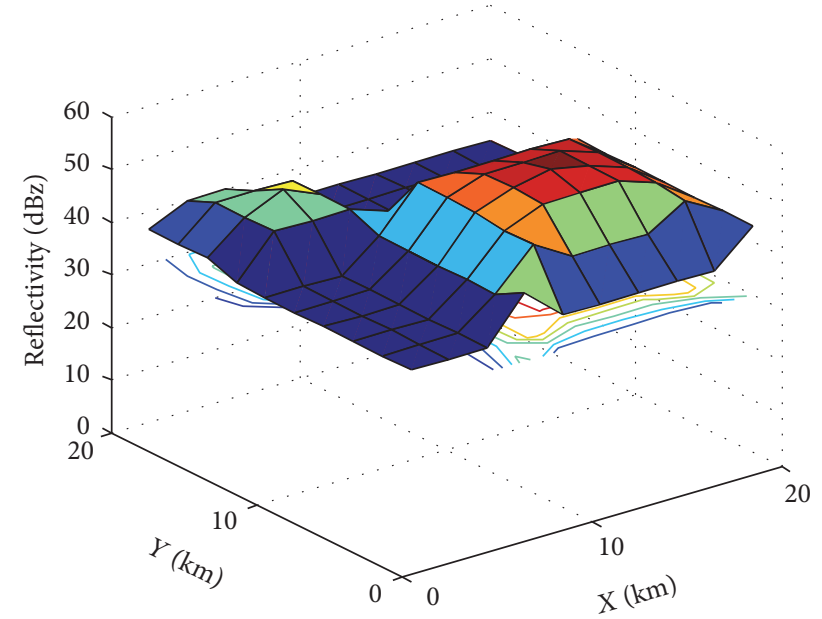

FIGURE 6: Initial field of radar reflectivity factor in the simulated area.

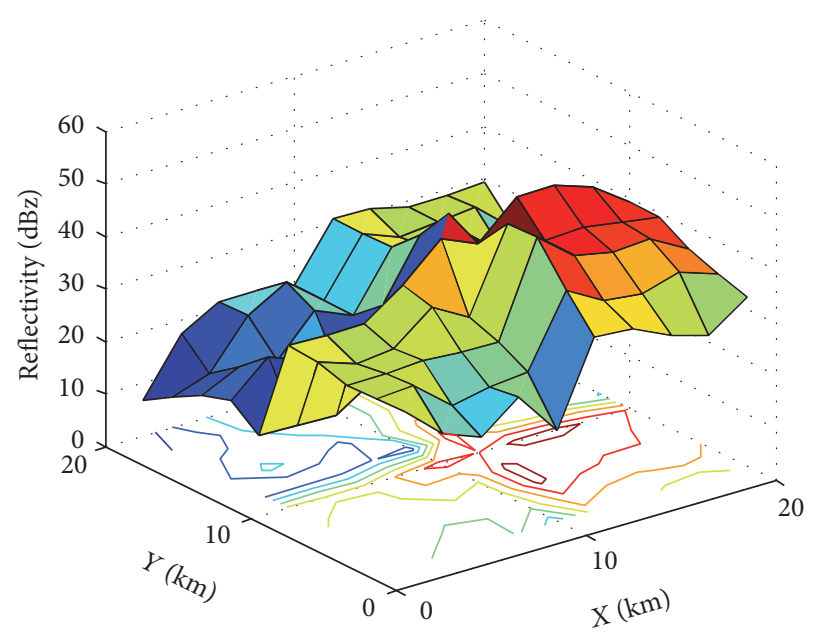

Figure 7: Observation field of radar reflectivity factor.

signal and microwave link signal were analyzed. After the attenuation of microwave links was matched with the weather radar data in the area, which was discretized, the model of the tomographic reconstruction was established for the attenuation coefficient of each grid cell, based on the multiplemicrowave-links network and using Simultaneous Iterative Reconstruction Technique. The established model of regional attenuation correction achieved the attenuation correction for the radar reflectivity factor, where the total attenuation value of the path was obtained by adding the distance between the radar sites and each grid.

A numerical simulation of regional attenuation correction $(20 \times 20 \mathrm{~km})$ was performed to verify the algorithm. There were 34 microwave links in the simulation area, and the microwave transmitting end performed the signal transmission at the frequency of $9.47 \mathrm{GHz}$ with vertical polarization. The results show that, compared with the uncorrected radar 


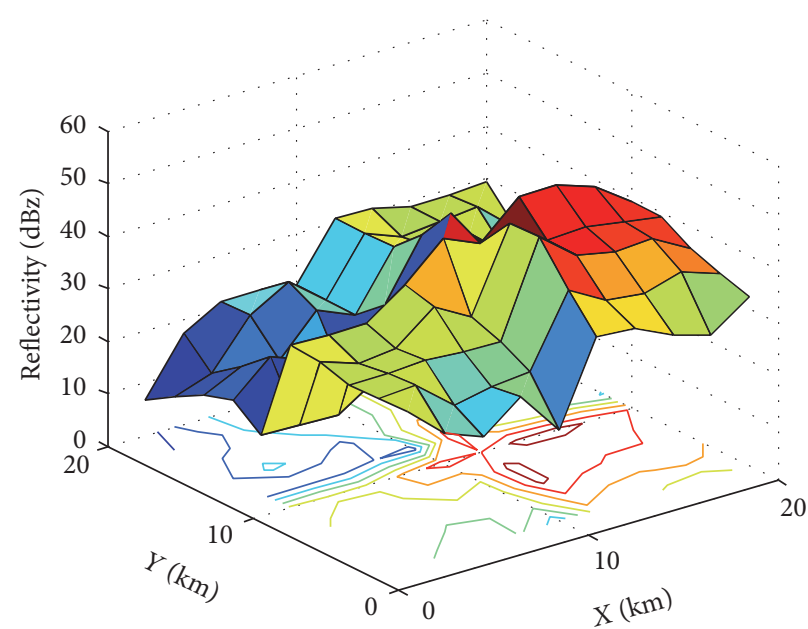

(a)

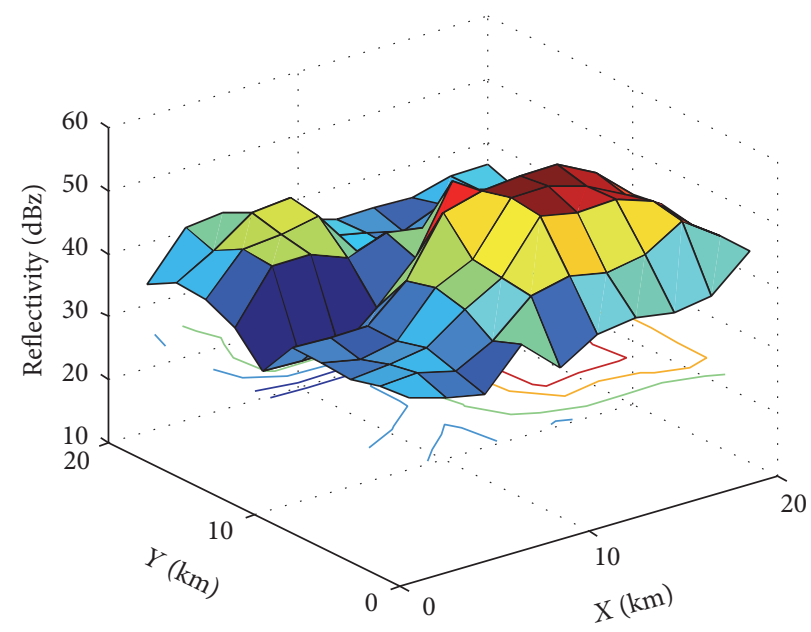

(b)

FIGURE 8: Radar reflectivity factor: (a) uncorrected; (b) corrected.

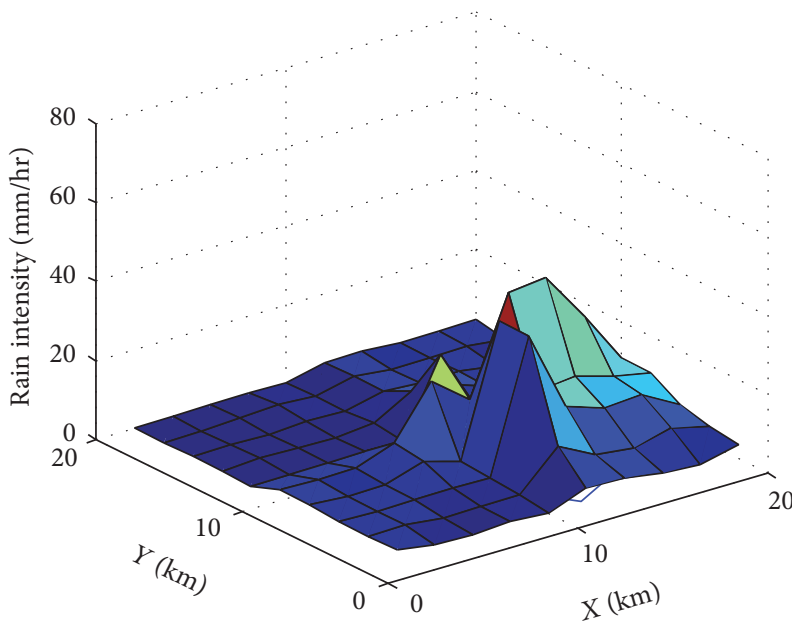

(a)

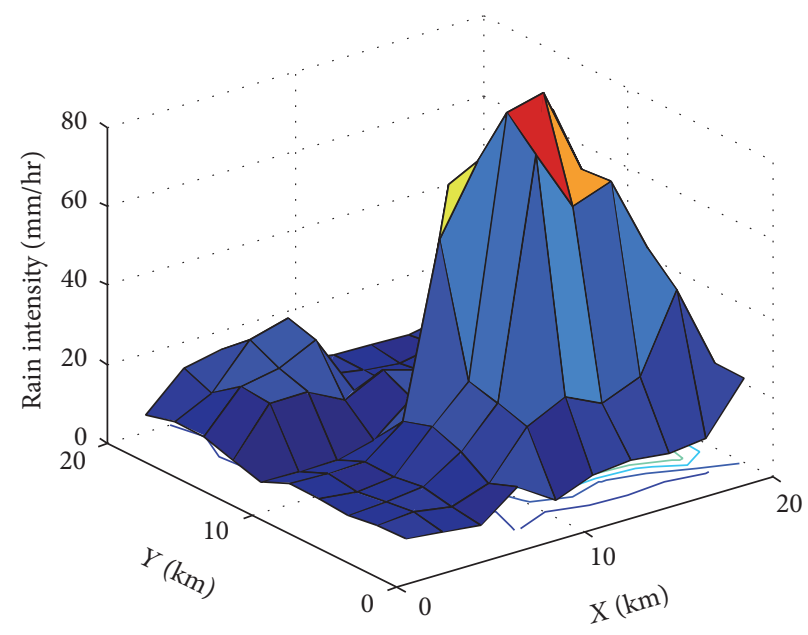

(b)

FIGURE 9: Rainfall intensity: (a) uncorrected; (b) corrected.

reflectivity and the uncorrected rainfall intensity, the accuracy of corrected radar reflectivity factor improved by $26.12 \%$, and the corrected rainfall intensity distribution improved by $51.85 \%$. Therefore, the region attenuation correction algorithm was validated.

The advantage of using regional attenuation correction for radar with a multiple-microwave-links network is that this technique enlarges the scope of correction when compared with the method of single-path correction. The method has important application value for radar attenuation correction effect and improves the precision of quantitative precipitation estimation by weather radar.

At present, commercial microwave links in cellular communication networks, electric power communication, maritime telecommunication, and microwave relay communication system have widely covered the urban and rural areas; on the basis of above equipment, there is no need to construct additional hardware; there is only a need to establish a network for signal acquisition from the existing microwave link devices; the economic cost is relatively low; therefore it has a unique advantage in the financial and engineering aspects. On the other hand, the regional attenuation correction method has a wider coverage and a higher spatial resolution than the single-point correction method, and the spatial representativeness is also much better. This paper has verified the feasibility and validity of above method. Aiming at the irregular distribution structures of the actual microwave links, we will investigate the attenuation coefficients reconstruction and regional attenuation correction under the condition of irregular topology network in the future.

\section{Conflicts of Interest}

The authors declare that there are no conflicts of interest regarding the publication of this paper. 


\section{Acknowledgments}

This work is supported by the Jiangsu Province Natural Science Foundation of China (Grant no. BK20150708) and National Natural Science Foundation of China (Grants nos. 41505135 and 41475020 ). The authors are very grateful to the team at the GTC Laboratory and the authors who work on the use of microwave links for rainfall measurement.

\section{References}

[1] B. Pauthier, B. Bois, T. Castel, D. Thévenin, C. C. Smith, and Y. Richard, "Mesoscale and local scale evaluations of quantitative precipitation estimates by weather radar products during a heavy rainfall event," Advances in Meteorology, vol. 2016, Article ID 6089319, 9 pages, 2016.

[2] X. C. Liu, T. C. Gao, J. Qin, and L. Liu, "Effects analysis of rainfall on microwave transmission characteristics," Acta Physica Sinica, vol. 59, no. 3, pp. 2156-2162, 2010.

[3] H. Lin and D. R. Lv, "Comparisons and combined uses of radar and radiometer in the remote sensing of rainfall distributions," Chinese Journal of Atmospheric Sciences, vol. 4, no. 1, pp. 30-39, 1980.

[4] S. Serrar, G. Delrieu, J. D. Creutin, and R. Uijlenhoet, "Mountain reference technique: use of mountain returns to calibrate weather radars operating at attenuating wavelengths," Journal of Geophysical Research: Atmospheres, vol. 105, no. 2, pp. 22812290, 2000.

[5] M. A. Perez, Improving precipitation estimates from dual wavelength radars [M.S. thesis], McGill University, Montreal, Canada, 2003.

[6] R. Meneghini, T. Iguchi, T. Kozu et al., "Use of the surface reference technique for path attenuation estimates from the TRMM precipitation radar," Journal of Applied Meteorology, vol. 39, no. 12, pp. 2053-2070, 2000.

[7] H. Messer, A. Zinevich, and P. Alpert, "Environmental monitoring by wireless communication networks," Science, vol. 312, no. 5774, p. 713, 2006.

[8] H. Leijnse, R. Uijlenhoet, and J. N. M. Stricker, "Hydrometeorological application of a microwave link: 2. Precipitation," Water Resources Research, vol. 43, no. 4, Article ID W04416, 2007.

[9] S. Krämer, H. Verworn, and A. Redder, "Microwave links A precipitation measurement method filling the gap between rain gauge and radar data," in Proceedings of the 6th International Workshop on Precipitation in Urban Areas, 2003.

[10] T. C. Gao, K. Song, X. C. Liu, M. Yin, L. Liu, and S. T. Jiang, "Research on the method and experiment of path rainfall intensity inversion using a microwave link," Acta Physica Sinica, vol. 64, no. 17, pp. 174301-174301, 2015.

[11] S. Krämer, H.-R. Verworn, and A. Redder, "Improvement of X-band radar rainfall estimates using a microwave link," Atmospheric Research, vol. 77, no. 1-4, pp. 278-299, 2005.

[12] A. R. Rahimi, A. R. Holt, G. J. G. Upton, S. Krämer, A. Redder, and H.-R. Verworn, "Attenuation calibration of an X-band weather radar using a microwave link," Journal of Atmospheric and Oceanic Technology, vol. 23, no. 3, pp. 395-405, 2006.

[13] S. Krämer and H. R. Verworn, "Improved C-band radar data processing for real time control of urban drainage systems," in Proceedings of the 11th International Conference on Urban Drainage, pp. 1-10, Hydro International, North Somerset, UK, 2008.
[14] R. J. Cummings, G. J. G. Upton, A. R. Holt, and M. Kitchen, "Using microwave links to adjust the radar rainfall field," Advances in Water Resources, vol. 32, no. 7, pp. 1003-1010, 2009.

[15] J. Radon, "On determination of functions by their integral values along certain multiplicities," Ber. der Sachische Akademie der Wissenschaften Leipzig, vol. 69, pp. 262-277, 1917.

[16] D. Giuli, A. Toccafondi, G. B. Gentili, and A. Freni, "Tomographic reconstruction of rainfall fields through microwave attenuation measurements," Journal of Applied Meteorology, vol. 30, no. 9, pp. 1323-1340, 1991.

[17] D. Giuli, L. Facheris, and S. Tanelli, "Microwave tomographic inversion technique based on stochastic approach for rainfall fields monitoring," IEEE Transactions on Geoscience and Remote Sensing, vol. 37, no. 5, pp. 2536-2555, 1999.

[18] Z. W. Zhao, M. G. Zhang, and Z. S. Wu, "Analytic specific attenuation model for rain for use in prediction methods," Journal of Infrared, Millimeter, and Terahertz Waves, vol. 22, no. 1, pp. 113-120, 2001. 

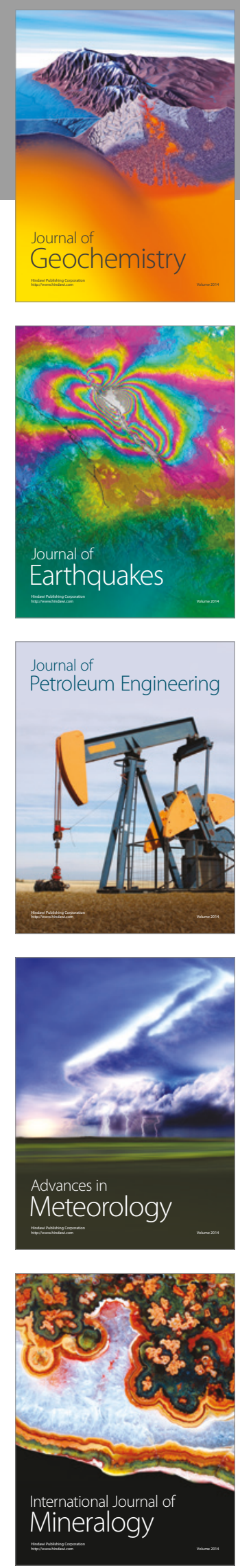
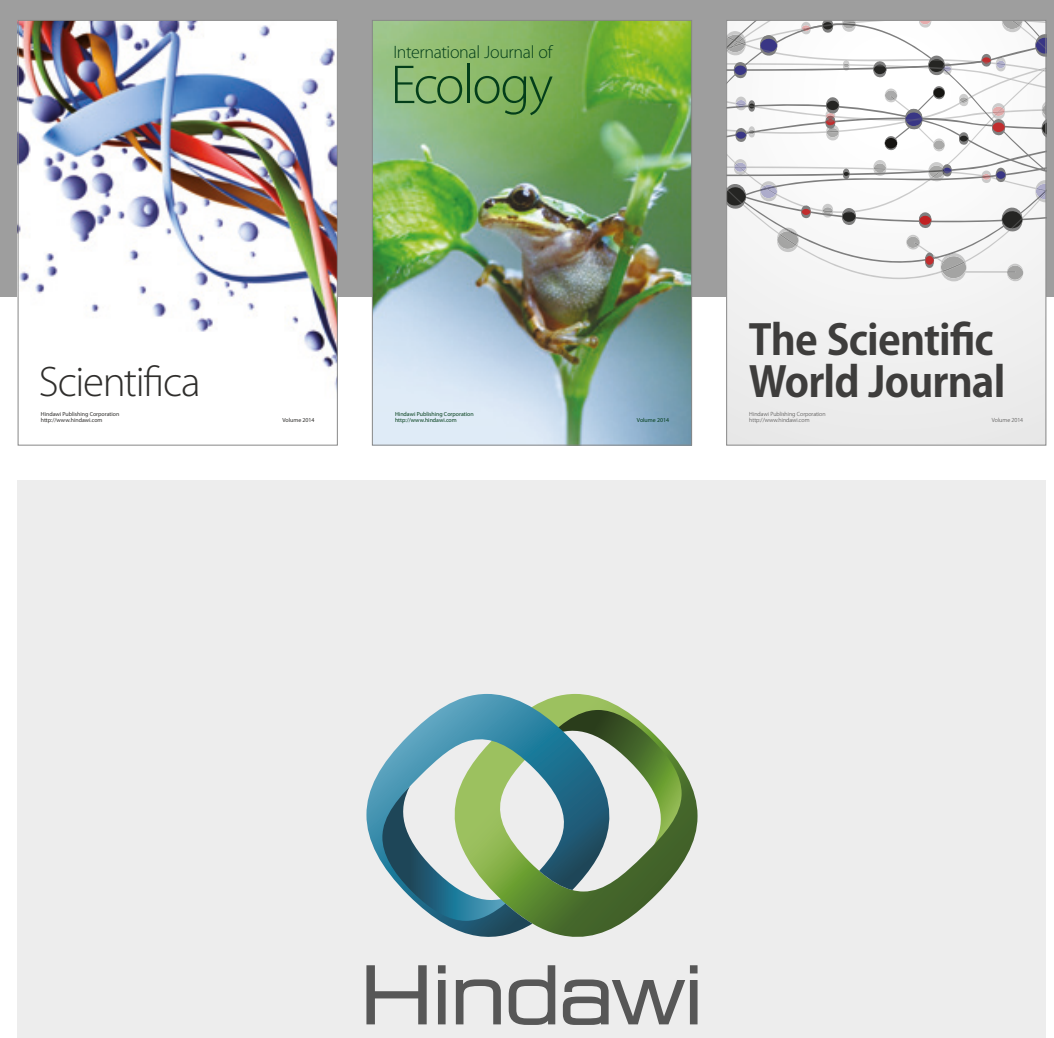

Submit your manuscripts at

https://www.hindawi.com
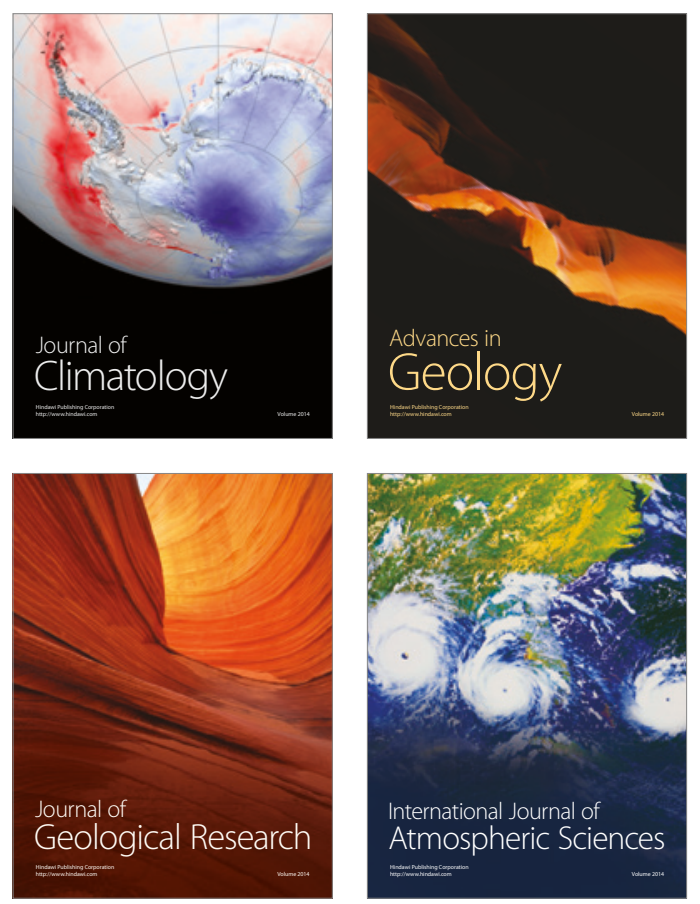

The Scientific

World Journal
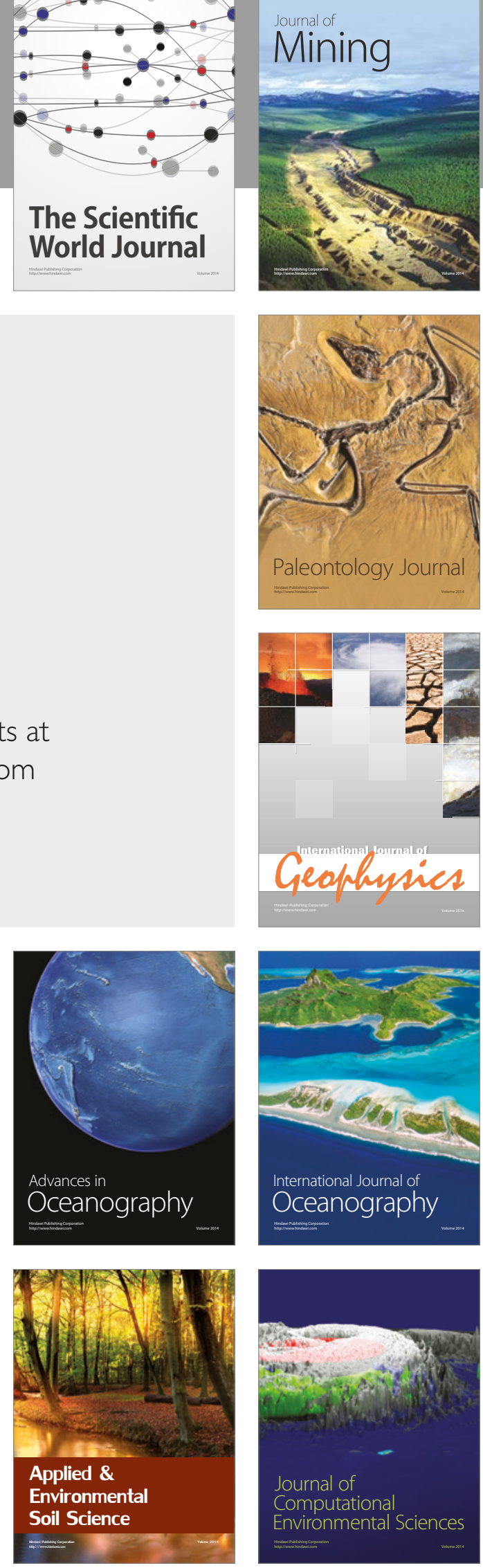